\title{
XLVIII. On the formation of emulsions, and the action of the bile in digestion
}

\section{Dr. G. Quincke}

To cite this article: Dr. G. Quincke (1879) XLVIII. On the formation of emulsions, and the action of the bile in digestion, Philosophical Magazine Series 5, 7:44, 301-313, DOI: $10.1080 / 14786447908639611$

To link to this article: http://dx.doi.org/10.1080/14786447908639611

曲 Published online: 13 May 2009.

Submit your article to this journal $\lceil\pi$

Џll Article views: 2

Q View related articles $\asymp$ 
LONDON, EDINBURGH, AND DUBLIN

\title{
PHILOSOPHICAL MAGAZINE
}

\author{
AND \\ JOURNAL OF SCIENCE.
}

[FIFTH SERIES.]

$M A Y 1879$.

XLVIII. On the Formation of Emulsions, and the Action of the Bile in Digestion. By Dr. G. QuINckE*.

1. Historical Review.

A $\mathrm{N}$ emulsion consists of a large number of small spherical A globules of fatty matter suspended in an aqueous liquid. Ordinary milk, for instance, is an emulsion. The smaller these fatty globules the larger is their surface in proportion to the mass, and the greater is the resistance they meet with in ascending through the specifically heavier surrounding fluid. The smaller the fatty globules the longer they remain suspended in the surrounding liquid, and the more perfect is the emulsion. The minute globules have a continual tendency to coalesce into larger ones; the less this tendency and the smaller the uniform velocity of the ascent of the globules in the surrounding aqueous liquid, the more permanent is the emulsion. And the smaller this velocity the less is the difference between the specific gravity of the emulsion and that of an actual solution of the fatty matter in the liquid†.

In chemists' shops an emulsion is made by diffusing mechanically (rubbing with a pestle in a porcelain mortar) the globules of an oil throughout a solution of gum arabic in water. In the process of digestion in the animal body the assimilation of the fats is initiated by the formation of an emulsion in the liquid contents of the intestine, and, as experience shows, faci-

* From a separate impression from Pflüger's Archiv für die gesammte Physiologie. Translated by J. F. Iselin, M.A.

† Camillo Bondy, Pogg. Ann. 1865, p. 323; E. Mach, ib. p. 329. Phil. Mag. S. 5. Vol. 7. No. 44. May $1879 . \quad 2 \mathrm{~B}$ 
litated by the bile. It is, on this account, as a physiological question that the development and durability of emulsions has more especially been studied.

W. Kühne (Physiologische Chemie,p.129, 1866) and Brücke (Wien. Sitzber. 1870, lxi. 2nd part, p. 363) were among the first to demonstrate the influence of the soaps developed in the small intestine on the formation of emulsions. The latter showed that rancid oil, containing free fatty acid, when agitated with dilute solution of the alkaline carbonates, produces a perfect emulsion with much greater rapidity than a neutral oil. He pointed out at the same time the important part played in the process of digestion by the free fatty acids, which, according to the discovery of Claude Bernard, are separated in the intestinal canal from the nentral fat by means of the pancreatic juice. More recently Johannes Gad* made the interesting observation that small quantities of oil, which contain free fatty acids, will form perfect emulsions by mere contact with alkaline solutions without the aid of external or mechanical means, such as agitating or stirring. This can be well shown by dropping a little codliver oil into a $\cdot 25$-per-cent. solution of soda.

From further investigation it appeared that the emulsifying power depends on the viscosity and acidity of the oil, on the concentration of the soda solution, and on the solubility in the surrounding fluid of the soap formed from the fatty acid. By adding common salt and bile to the alkaline liquid, this tendency to dissolve is so far corrected that the facilities for the production of a good emulsion aro much increased. With castor-oil, which is more viscous than other oils, the formation of an emulsion was not observed. When the conditions for the emulsion-formation were present, the surface of the drop of oil threw off at once a milky substance into the surrounding liquid; the drop formed protuberances at the side, and exhibited alterations in form and movements which possess remarkable similarity to those of the Amcba. Smaller oil-globules then split off; and these partly gave rise to the further production of emulsion. Under the microscope with a low power the vioinity of the drop was seen to be the scene of brisk action; the particles producing turbidity in the fluid were observed to fly off in rapid gyrations from the surface of the drop, sometimes retrorning to it again. The remainder of the oil could not by mechanical means be converted into emulsion in the same liquid.

Gad recognizes therefore, quite correctly, the conditions

* Du Bois-Reymond's Avchiv für Anatomie und Physiologie, 1878, pp. 181-205. 
for emulsion-development, not in the soap dissolved in the liquid, but in that which forms more or less rapidly on the surface of the oil, where, in fact, the fatty acids contained in the oil come into contact with the alkaline liquid. He then proceeds to observe that when the soap that has been formed under the given conditions is soluble in the surrounding liquid, it is by diffusion carried off radially from the point where it is developed, while inside the globule the equilibrium of solubility is maintained by diffusion of the fatty acid outwards towards the periphery. If the soap be so rapidly formed and removed that the fatty acid by diffusion towards the periphery cannot make up the deficiency, the outer edge of the globule will alter its shape, and there will be thrown off smaller fatty particles which are not enclosed in a soapy membrane. But when the rapidity with which the soap is formed reaches a certain point, the latter will not be dissolved by the surrounding liquid, and the oil-globule will be enveloped by a soapy membrane. The development and displacement of the soapy matter will give rise to a change of volume in the liquid ; and this in its turn will cause the breaking-away of small fatty particles, each of which is enclosed in a film of soap. Should there be from any cause an irregularity in the course of the formation of the soapy membranes, it will occasion Amobalike movements, the protuberances being extended at the points where the membrane takes a longer time to thicken.

To these theoretical views, however, I am unable to assent, because it appears to me to be highly improbable that mere diffusion currents can produce movements so energetic as those that are observed in the production of emulsions. In the following pages I shall endeavour to prove that the formation of an emulsion depends essentially on the existence of thin scales of soap solution dispersed over the common surface of the oil and the liquid, and also that the so-called Amoba-like movements depend on the same cause.

\section{The Bounding Surfaces of Liquids in Contact with Air and with Water.}

The surface of every liquid, whether the same be bounded by air or by another liquid, has a tendency to become as small as possible, or, as it is commonly termed, has a certain tension. The magnitude of this tension, which may be likened to that of a cloth, or to that of the envelope of a caoutchouc balloon, or of an inflated pig's bladder, is measured by the force (in milligrammes) exerted on a strip of the surface one millimetre broad. The tension of the surface of a liquid bounded by air is at the same time a measure of the cohesion 


\section{Dr. G. Quincke on the Formation of Emalsions,}

of the liquid, and is generally found by multiplying together the height at which the fluid will stand in a capillary glass tube, the radius of that tube, and half the specific gravity of the fluid.

In consequence, however, of the difficulty of thoroughly moistening the interior of the tube with the fluid*, this method is not quite accurate. It is a better way to determine the tension by measuring the vertical distance between the apse and vertex of a large flat bubble of air lying in the fluid underneath a horizontal glass plate; the square of this distance into half the specific gravity of the fluid gives the surfacetension directly, independent of the nature of the plate under which the bubble lies. The shape of such a bubble is the same as that of an inverted dewdrop in air. By forming a flat bubble of some other fluid in the heavier fluid (for example a bubble of oil in water) the surface-tension of the common surface of the two fluids may be found in a similar way-for instance, in this case by multiplying the square of the vertical distance between the bubble's apse and vertex into half the difference of the specific gravities of oil and water.

We find by this method the tension at the common surface of air and water to be $=8.25 \mathrm{mgr}$, of air and olive oil $=3.76$ mgr., of water and olive-oil $=2.30 \mathrm{mgr}$. Other fatty oils, rape-oil, almond-oil, castor-oil, cod-liver oil, \&c., give similar results. Fluids which mix with water in all proportions, like alcohol or dilute salt solutions, form nẹther bubble nor globule in water; the tension of the common surfaces of such fluids and water is $=0 \dagger$.

\section{The Dispersion of Soap Solutions and of other Fluids over the common Surface of Oil and Water.}

When some other fluid is applied to an air-bubble in water, and it disperses itself over the surface of the bubble, the vertical distance between the vertex and apse of the latter is diminished; in other words, the tension at the common surface of air and water has been reduced. For instance, when olive oil is dispersed over the common surface of air and water, the air-bubble is coated with a thin film of oil ; and the surfacetension is now compounded of the tension at the surface of air and oil, and of the tension at the surface of oil and water.

* Compare G. Quincke on the Cohesion of the Solutions of the Salts, Poggendorft's Annalen, clx. p. 369, 1877.

$\dagger$ The more detailed explanation of these pbysical laws, as well as of the theory of the dispersion of one fluid substance over the surface of another, I have given with mathematical and experimental illustrations in Poggendorff's Annalen, cxxxix. p. 1, 1870; ib. clx. pp. 387 and 560, 1877 ; and in Wiedemann's Annalen, ii. p. 144, 1877. 
In a similar way a fluid like soap-solution will spread itself over the surface of a flat oil-bubble in water. At the common surface of soap-solution and olive-oil the tension is $=0 \cdot 36$ mgr.; but at the common surface of soap-solution and water the tension is $=0$; and as the original surface-tension of oliveoil and water is $2.3 \mathrm{mgr}$, this tension has been reduced to the extent of 84 per cent. by the dispersion of the solution of soap. It will be found that the oil-bubble itself has been made considerably flatter and broader by the dispersion.

The alteration in form of an air- or oil-bubble increases with the thickness of the film of the applied fluid, and attains its maximum value when that thickness is more than 0001 millim., or one-fifth of the length of a mean light-wave in air. The thickness of such a film therefore cannot be recognized by even our best microscopes; for they only detect a distance equal to half a wave-length*. As exceedingly dilute solutions of soap (1 per cent. and less) are able to produce this effect, we observe that an excessively small quantity of solid soap, which in any other way we could scarcely detect, is sufficient to cause this phenomenon of dispersion. And this dispersion takcs place with great rapidity; a drop of olive-oil will spread itself over the surface of still water in a single second, and cover a space several metres in diameter.

Similar to the action of soap-solution is that of diluted ox-gall, or of a fluid obtained from the action of a dilute solution of soda on the free fatty acid contained in oil. In a rectangular glass trough a horizontal plane glass plate was suspended, the latter being pierced in the centre with a hole of 2 millims. diameter. The trough was then filled with water up to the lower surface of the glass plate; and through the bole, by means of a pipette, a globule of oil was introduced underneath the glass plate. With a second pipette a few drops of a $\cdot 25$-per-cent. solution of soda were introduced; and this immediately sank in the oil and retreated to the lowest side of the globule. The soda formed with the free fatty acid a soap, which then enveloped the drops of soda solution lying in the oil with a more or less thick whitish membrane. If this membrane be then broken up by agitation, or by its coming into contact with and dissolving in the adjacent water, the solution of soap or a mixed solution of soap and soda spreads itself over the surface of the globule of oil, and the latter becomes flatter and broader.

* The proof of these propositions will be found in my treatise "On the Distance at which the Molecular Force of Capillarity can act," Poggendorft's Annaten, cxxxvii. p. 402, 1869 ; also "On the Edge-angle and Dispersion of Fluids on Solid Bodies," Wiedemann's Annalen, ii. p. 177, 1877 (Phil. Mag. [5] vol. v. pp. 321, 415). 
Simultaneously with this dispersion of the soap-solution, a current is set up from the interior of the fluid towards the centre of dispersion, and continued onwards from its surface. This current is much stronger in the tenacious oil than in the water. The bubble becomes for a short time concave at its vertex ; isolated particles of oil are torn away by the current from the main body, and form spherical globules in the surrounding aqueous fluid; and vortices are produced in the oil similar to those caused by blowing a stream of air through a vertical and narrow glass tube on to a free and plane surface of oil. If a layer of oil of from 5 to 10 millimetres thickness be poured on water, and alcohol allowed to spread itself over either the upper or under surface of the oil, those parts of the oil which are opposite to the centre of dispersion will move towards that centre; the layer of oil may even be pierced by this means, so that air and water come into contact. Excessive rapidity of dispersion in the applied fluid, or too much tenacity in the oil, will impede the expulsion of the oil particles or the piercing of the oil layer just as much as sluggishness in the dispersion or too great ductility in the oil.

\section{Explanation of the Spontaneous Formation of Emulsions by means of Dispersion.}

By the phenomena described in the above paragraphs we are now able to explain the formation of an emulsion.

When a solution of soda and an oil come into contact, a solid suap is formed by the action of the free fatty acid of the oil. Gradually a portion of this soap dissolves in the adjoining aqueous fluid. So soon as the fluid solution of soap comes into contact with the oil, it spreads itself over the bounding surface of the oil and aqueous fluid, and carries with it the undissolved particles of soap with any adhering oil-globules. In this way there are detached from the oil and borne into the adjoining fluid a number of filaments, which, possessing the tendency to assume the shape of bodies of least surface, are converted into larger or smaller globules of a spherical form, just as a jet of water in the air breaks up into larger and smaller drops. To a certain extent this conversion of the oil into globules will be retarded by the solid and fluid soap already present or newly formed; and then the length of the filaments will be increased, or the size of the nascent globules diminished. By the original dispersion, however, fresh oil particles will be brought into contact with the soda solution, and after a time the newly formed solid soap is again dissolved and a second dispersion occurs. Similar periodic dispersions of oil on the 
surface of still water were observed and described by me nine years ago (Poggendorff's Annalen, cxxxix. p. 76, 1870).

The periodic dispersions of soap solution over the common surface of the oil and an aqueous fluid, which are not simultaneous at all points of the oil-surface, will be found to be connected with vortex-movements in the interior of both fluids, and to draw the oil more especially towards the dispersion-centre. This is the cause of the Amoebc-like movements at the edge of the main mass, while the detached particles of oil form the emulsion. In fact, under the microscope, not only the aqueous fluid can be seen, but also, and more conspicuously, the oil in the vicinity of the common surface, in a state of gyration.

When the oil possesses a tolerable degree of toughness, and the dispersive force at the common surface of the two fluids is moderately great, the vortex-movements and the detached oil particles will be very numerous, and may be produced, as indeed follows from the dispersion theory (see above, 3, p. 305), by means of films of diluted soap solution not excoeding in thickness a few millionths of a millimetre. A very minute quantity of soapy matter is therefore sufficient to produce the appearances in question. The free fatty acid necessary for the formation of this soap is nearly always present in oil, and reaches the surface of the latter by means of diffusion. It ean be produced in the fluid contents of the intestine by means of the pancreatic juice, and in the open air by the action of carbonic acid on the neutral alkaline oleates*. If the soap be formed too rapidly, the common surface of the oil and the aqueous fluid is coated with a film of solid soap. Hence the oil-surface becomes immobile, and the dispersion and its consequences are impeded or are altogether wanting-just as, in Leidenfrost's experiment, when water is brought into contact with red-hot metal the formation of steam is impeded or does not occur at all.

In the same way the consequences of the dispersion, the formation of emulsion and the movements of the particles of oil, will be prevented, if only a very small quantity of soap be formed, or if the soap be dissolved too rapidly by the surrounding fluid. Every, even the minutest, particle of soap will then immediately be diffused in a solution, and the dispersive energy will not be sufficient to produce the vortex-movements in the oil, and to detach the particles of that substance. If a mill-brook be allowed to trickle in a small strcam over the water-wheel, the latter will not be set in motion; but by periodically damming it back a small body of water can be made with advantage to produce mechanical work.

$$
\text { * Compare Heintz, Zoochemie, p. } 439 .
$$




\section{Solid and Liquid Films at the common Surface of two Fluids. Castor-oil.}

For the reason last stated, castor-oil will not freely form an emulsion.

When a drop of a fixed oil is allowed to fall at as small a distance as possible from the end of a glass rod onto a layer of a dilute solntion of soda which is only a few millimetres in thickness, and is contained in a watch-glass of from 50 to 100 millimetres diameter, the oil will generally, as explained more fully above (in 3), disperse over the surface of the liquid; for the surface-tension of the more perfect fluid is greater than that of the oil. Shortly afterwards soap is formed and dissolved in the aqueous liquid, the tension of the free surface of the aqueous liquid becomes considerably less, and the oil contracts again into a lenticular-shaped drop. On its lower surface the drop of oil is coated with a film of soap, which may consist of a thin membrane either of liquid solution of soap or of solid soap. The latter is well seen when a number of solid particles of soap lying close to each other form a whitish matted film, as in the case of cod-liver oil in a from $\frac{1}{4}$ to 2 -per-cent. solntion of soda.

If the soapy membrane be liquid, the oil-drop assumes a spherical form, which it will retain even when the liquid in the watch-glass has a rocking or rolling motion communicated to it. If, on the other band, the membrane be solid, the drop under the rocking motion takes a cylindrical shape, which disappears more or less slowly in proportion as the membrane is thicker or thinner. This was the case with olive, almond, and cod-liver oils in a 0.06 -per-cent. solution of soda, and of almond-oil in a 0.25-per-cent. solution. A similar appearance is observed in the case of a drop of mercury in a clean watchglass, which, when coated with a thin layer of oil, retains its spherical form, notwithstanding its being rolled about on the glass. If, however, the mercury contain a small quantity of a solid metal, as, for example, lead, which forms in contact with the air a film of solid oxide, the drop will become cylindrical under the influence of a rocking motion.

With castor-oil in dilute solutions of soda, I have observed atter the lapse of some hours a trace of a whitish precipitate at the lower surface of the oil-drop; but the latter is always in the shape of a sphere. When shaken, the drop becomes larger for a few minutes, probably because the agitation causes a little soap to be thrown from the interior of the drop to its surface, where it is dispersed. But so soon as this soap is dissolved in the aqueous fluid, and the common surface of the two liquids 
returns to its former condition, the drop of oil will also assume its original shape.

\section{The Influence of Bile.}

For the bile that I employed in my experiments I am indebted to the kindness of my colleague W. Kühne; it was prepared by evaporating to dryness over the water-bath an alcoholic solution of crystallized ox-gall, and dissolving the residue in water.

If some solution of bile be added to the fluid in which floats a drop of olive, almond, or cod-liver oil coated with a solid soap membrane, this membrane will be dissolved ; the oil-drop assumes the spherical form, and retains it after agitation. On this ability of bile to transform a solid into a fluid soap, or into a soap-solution, seems to depend its property of promoting the assimilation of fat in the animal body. Solid particles of soap on the surface of an oil-drop prevent any change of form in that surface, and thus diminish its ability to pass through animal membranes. This hindrance is removed so soon as the drop is coated with a fluid membrane. Bile has the property of promoting the spontaneous formation of an emulsion when the solid soap at the common surface of the oil and the sodasolution dissolves slowly; but it counteracts the emulsion-formation when it converts the solid soap into a liquid too rapidly. Both of those phenomena were observed by Gad.

According to the views of C. A. von Wistingshausen*, the bile, drawn through the walls of the lacteals, is accompanied by the adhering particles of fatty matter; but, as it seems to me, this theory has not up to the present been proved. The same observer claims to have found that oliveoil will rise higher in capillary tubes moistened with various liquids when those liquids contain in solution salts of the biliary acids. For moistening the tubes, water and dilute solution of potash were used, with or without the addition of alloumen.

Unfortunately we are unable to gather from the descriptions of these experiments in what way the capillary glass tubes were moistened with the liquid. If the oil in rising drives before it a continuous layer or column of the liquid, my own experience is, contrary to that of von Wistingshausen, that oil will rise higher in capillary tubes wetted with water than in those moistened with solutions of bile of different degrees of concentration. In the presence of potash it may behave differently; the solid soap

* Compare J. Steiner on C. A. ron Wistingshausen's "Researches on the Action of the Bile in the Absorption by Endosmose of the Neutral Fats," Du Bois-Reymond's Archiv, 1873, p. 139. 
at the common surface of the oil and aqueous liquid renders the fluid column in the capillary tube less mobile, and therefore prevents the oil from rising so high. But by the addition of the solution of bile the soap is rendered soluble, the friction in the interior of the tube is diminished, and consequently the height to which the oil rises is indirectly increased.

It has been often stated (compare Gad, Du Bois's Arch. 1878, p. 202) that from experiments of this kind we obtain an idea of what takes place on a very much reduced scale with the diffusion of fluids through the capillaries of the animal membrane. But it must be remembered that the ascending action in capillary tubes depends on the nature of two surfaces-one the common surface of water or aqueous fluid and air, the other that of the same substance and oil. Now the first of these, on account of its greater surface-tension, has a much more decided influence on the capillary height than the second. And it also happens that the height varies with the curvature of the common surface of the oil and the fluid; in glass tubes the convex side of the common surface is generally turned towards the aqueous liquid. But this curvature will vary considerably in the same tube, and depends materially on the edgo-angle of the common surface with the wall of the tube; and the edge-angle has very different values in the case of tubes of glass and of those of the animal membranes. Now, under normal circumstances the surfaces of the fluids of the animal body are in contact neither with glass nor with air; conseguently no opinion can be formed of the operations of diftusion in the animal body by the measurement of capillary heights, which depend essentially on the common surfaces of fluids with glass and air. A closer insight into these phenomena of diffusion can only be obtained by an investigation of the physical and chemical actions at the common surface of one kind of fluid with another, or with the animal membrane itself.

\section{Permanence of Emulsions. Froth.}

J. Plateau (Mém. de Brux. xxxvii. p. 3, 1868) first showed that many liquids appear to possess greater viscidity on their outer than on their inner surface. More recently I myself (Poggendorff's Annalen, exxxix. p. 71, 1870), as well as Marangoni (Cimento [2], v.-vi. p. 239, 1872), found that a free liquid surface in contact with the air becomes less mobile as soon as a thin film of some extraneous fluid is diffused over it. The same thing occurs when an extraneous liquid (solution of soap) is diffused over the common surface of the other liquids (oil and water). 
The immobility or permanence of the common surface thas modified is explained by the fact that each opening in the film of extraneous liquid is immediately closed by the molecular forces; the surface-tension of the pure liquid in the opening is greater than that of the rest of the film contaminated by contact with the extraneous liquid. The extraneous film may also consist of a solid body, provided that it be not completely solid, but to some extent mobile. This follows from the above-described (under 5) phenomena and properties of the common surfaces of liquids and solid bodies (compare Wiedemann's Annalen, ii. p. 145,1877$)$.

In emulsions of the fixed oils in a solution of soda, the thin film of soap, each aperture in which is closed again by molecular force, prevents the oil-globules from coalescing. In emulsions of gum as prepared by the druggists, each minute oil-globule is separated from the aqueous fluid by a film of the solution of gum; for at their common surface with gum-solution the fixed oils have a less surface-tension than at that with water; this property I have proved by special measurements, as I shall have occasion to explain at greater length in another place. The longer the gum-solution remains attached to the surface of the oil, the firmer it will adhere.

- When mercury is agitated with water and olive-oil, a viscous mass of a grey colour is formed: this is an emulsion of mercury, consisting of a number of very small globules of- the metal, each of which is coated with a film of oil kept adhering to it by molecular force. A fissure in the oil-film of one millimetre breadth will reunite with a force which, according to my own measurements, amounts to $6.09 \mathrm{mgr}$., and is therefore comparatively great. In fact, an emulsion of this kind will endure for months. The addition of an acid breaks up the oil-film, and consequently destroys the emulsion. So-called grey ointment is an emulsion of mercury in a highly viscous fat; its permanence increases with keeping, in consequence of the mercury forming with the rancid fat a compound (mercurial soap?) which appears to diffuse itself over the common surface of the two substances.

Froth which may be seen in a solution of soap or in beer, is an emulsion of air in an aqueous fluid. Its permanence is greater in proportion as the original surface-tension of the pure liquid is decreased by an extraneous film on the free surface that is bounded by air. Homogeneous fluids containing no heterogeneous substance do not form a froth; it can be obtained from fatty but not from pure water. The force with which a rent of the breadth of a millimetre in the film of extraneous fluid on water tends to reunite, in the case of soap- 
solution is $5.58 \mathrm{mgr}$., of albumen $2.40 \mathrm{mgr}$, and of a tenper-cent. solution of tannic acid $2 \cdot 88 \mathrm{mgr}$.

With organic fluid substances like albumen and solution of tannic acid, a thin membrane of the fixed substance seems to form on the surface of the air-bubbles; for they show angular protuberances, and the surface becomes less mobile; this immobile membrane promotes the durability of the emulsion. A permanent froth of this kind made with white of egg is well known in the kitchen.

With very volatile liquids, or such as are easily soluble in the original aqueous fluid, such as alcohol and ether, the froth soon subsides; for the filn of extraneous liquid on the surface of the water rapidly disappears, owing either to evaporation or solution. The froth of beer is broken up by a small quantity of ether; for the tension of the common surface of that substance and air is very slight, and the surface itself tears under the dispersion of the fluid laminx forming the foam, in a similar way as in the experiments on the dispersion of soap-solution over the common surface of oil and water, described above (under 3 ), where particles are split off from the main mass of oil.

\section{Conclusions.}

(1) A solution of soap disperses itself over the common surface of oil and water.

(2) This dispersion causes, in the interior both of the oil and the surrounding fluid, eddies or vortex-movements, by which particles of the oil are isolated or detached, and are drawn into the surrounding fluid, where they form small globules.

(3) Very minute quantities of soap, so small that they cannot even be recognized by the microscope, are enough to produce this dispersion-phenomenon and the movements in the whole body of oil caused by it.

(4) Fixed oils containing free fatty acid form in a weak solution.of soda a solid soap, which dissolves in the surrounding fluid and is dispersed over the surface of the oil.

(5) With a certain concentration of the solution of soda, and a certain solubility of the soap that is formed, the dispersion occurs at certain intervals, and detaches a large number of the small oil-globules. This explains the spontaneous formation of an emulsion observed by Joh. Gad, and the Amoebalike movements of the oil-globules in dilute solution of soda.

(6) The globules of oil are coated with a thin film of soap, either solid or dissolved in water; and this film, by molecular action, causes the oil-surface to be less mobile, prevents the 
globules from coalescing, and materially promotes the durability of the emulsion.

(7) Apothecaries' emulsions consist of oil-globules coated with a thin film of gum, which is retained at the oil-surface by molecular force, and prevents the small globules from coalescing to form drops of oil.

(8) In the case of castor-oil an emulsion cannot be readily formed, because the soap produced by the contact of the oil with the soda-solution is highly soluble.

(9) Bile facilitates the solution of the solid soap, and on that account promotes the development of emulsion, in the fluid contents of the intestine, or under certain circumstances may impede it. But the mobility of the oil-surface is increased by the bile.

(10) From the height of the ascent in capillary tubes, or from the behaviour of Huids at their common surface with air, no conclusions can be drawn as to the phenomena that will oceur at their common surface with other liquids or with solid bodies.

(11) Froth is an emulsion of air instead of one of oil. Its permanence depends on the same physical conditions as that of oil emulsions.

XLIX. On the Photographic Method of Registering Absorption-Spectra, and its Application to Solar Physics. By Capt. W. DE W. AbNeY, R.E., F.R.S.*

$r$ THERE are certain difficulties in registering the visible absorption-spectra as observed, dependent on the eye of the observer, and on his power of representing correctly what he sees; and it is owing to these deficiencies that curious mistakes have been made in endeavouring to draw absorption-phenomena. Up to the present time it has been, comparatively speaking, useless to attempt such registration by means of photography, owing to the fact that merely one part of the spectrum was impressionable by the silver salts employed as a sensitive medium. Since my discovery that silver bromide could be prepared in such a molecular state as to be sensitive to the whole spectrum (visible, ultra violet, and ultra red $\dagger$ ), the difficulty in the employment of photography is done away with; and it should be taken into use as much as possible, so as to eliminate the errors of eye-observations. A natural objection would arise at first sight, viz. that for the

* Communicated by the Physical Society.

$\dagger$ Except those radiations of low amplitude and large wave-length which are emitted by bodies at ordinary temperatures. 